\title{
INFECÇÃO DE SÍTIO CIRÚRGICO EM PACIENTES SUBMETIDOS A ARTROPLASTIAS DE QUADRIL ${ }^{1}$
}

\author{
Flávia Falci Ercole ${ }^{2}$ \\ Tânia Couto Machado Chianca ${ }^{3}$
}

Ercole FF, Chianca TCM. Infecção de sítio cirúrgico em pacientes submetidos a artroplastias de quadril. Rev Latino-am Enfermagem 2002 março-abril; 10(2):157-65.

Estudo epidemiológico, tipo coorte, não concorrente, sobre infecções de sítio cirúrgico conseqüentes às artroplastias de quadril, baseado em informações contidas em 305 prontuários de pacientes operados na Santa Casa de Belo Horizonte. Os objetivos foram determinar a taxa de incidência de infecção de sítio cirúrgico; verificar associação entre a infecção e fatores de risco; determinar o tempo de manifestação da infecção de sítio cirúrgico e identificar os microorganismos envolvidos. O microorganismo prevalente foi o Staphylococcus aureus. Encontrouse uma taxa de incidência de infecção de sítio cirúrgico acumulada de 8,5\%. Os fatores de risco para infecção foram: condição clínica do paciente, tempo de internação pré e pós-operatório, tipo de anestesia, modalidade cirúrgica e unidade de internação.

DESCRITORES: epidemiologia, infecção hospitalar, próteses e implantes

\section{SURGICAL SITE INFECTION IN PATIENTS SUBMITED TO HIP ARTHROPLASTY}

This is an epidemiological non concurrent cohort study on surgical site infections after hip arthroplasties, based on the information contained in 305 records of patients operated at the "Santa Casa de Belo Horizonte" Hospital. The goals were to determine the incidence rates of surgical site infection in patients, aiming at verifying the association between the surgical site infection and some risk factors and at identifying the time for the manifestation of surgical site infection and the microorganisms involved. The prevalent microorganism was the Staphylococcus aureus. A 8,5\% accumulated incidence rate of surgical site infection was found. The risks factors associated to infection were patient's clinical condition (ASA); pre and post-operative hospitalization time; type of surgical procedure; anesthesia and hospitalization unit.

DESCRIPTORS: epidemiology, hospital infections, prosthesis and devices

\section{INFECCIONES DEL SITIO QUIRÚRGICO EN PACIENTES QUE FUERON SOMETIDOS A ARTROPLASTIA DE CADERA}

Estudio epidemiológico, tipo cohorte, no concurrente, de infecciones del sitio quirúrgico después de la artroplástia de cadera, con base en informaciones contenidas en 305 historias clínicas de pacientes. Estos pacientes fueron operados en la Santa Casa de Belo Horizonte. Los objetivos fueron: determinar la tasa de incidencia de las infecciones del sitio quirúrgico; verificar la asociación entre las infecciones y algunos factores de riesgo; identificar el tiempo para la manifestación de la infecciones y los microorganismos comprometidos. El microorganismo prevaleciente fue el Staphylococcus aureus. Se encontró una tasa de infección de sitio quirúrgico de 8,5\%. Los factores de riesgos asociados a la infección fueron la condición clínica del paciente (ASA); tiempo de hospitalización pré y postoperatoria; tipo de procedimiento quirúrgico; tipo de anestesia, tipo de cirugía y unidad de hospitalización.

DESCRIPTORES: epidemiología, infeccioneshospitalaria, prótesis e implantes

\footnotetext{
${ }^{1}$ Este artigo é parte da dissertação de mestrado de Ercole, F.F.: "Aspectos epidemiológicos da infecção de sítio cirúrgico em pacientes submetidos a artroplastias de quadril em um hospital de grande porte de Belo Horizonte-Minas Gerais; ${ }^{2}$ Enfermeira, Mestre em Enfermagem, Doutoranda do Programa de Pós-Graduação do Departamento de Parasitologia do Instituto de Ciências Biológicas da Universidade Federal de Minas Gerais; ${ }^{3}$ Enfermeira, Doutor em Enfermagem, Professor Adjunto, Pós-Doutoranda na Universidade de lowa, EUA, e-mail: grey@mat.ufmg.br. Escola de Enfermagem da Universidade Federal de Minas Gerais
} 
INTRODUÇÃO

A preocupação com o problema da infecção hospitalar (IH) sempre esteve presente no decorrer de nossa experiência profissional, a maior parte dela referente ao trabalho em Central de Material Esterilizado e em Unidades de Centro Cirúrgico.

Sabe-se que fatores do paciente, ambiente, membros da equipe e materiais têm sido relacionados à incidência de infecção hospitalar em pacientes cirúrgicos ${ }^{(1-2)}$ e preocupam-nos, à medida que temos, enquanto enfermeiras de sala de operações, compromissos e responsabilidades com o controle das infecções hospitalares.

Nossa atenção voltou-se, principalmente, para as cirurgias de artroplastia de quadril, uma vez que, nestas, são utilizados materiais de implante não orgânicos que, por si só, possuem um risco de infecção elevado. Além disso, uma vez retirada a prótese, ela não poderá ser reaproveitada, causando, além de grandes transtornos ao paciente, acréscimo no tempo de internação e elevação nos custos hospitalares.

Ressalta-se a importância do pessoal de enfermagem no controle das infecções hospitalares, bem como o seu papel crítico como vetor de sua transmissão, por meio das mãos, no contato contínuo com os pacientes ${ }^{(3)}$. 0 enfermeiro, por meio da educação continuada, repassa conhecimento e informações técnicas ao restante da equipe e a outros profissionais da saúde, contribuindo, significativamente, para a minimização das infecções hospitalares e suas graves conseqüências.

A taxa de infecção de sítio cirúrgico (ISC) nas cirurgias de artroplastias de quadril na Santa Casa de Belo Horizonte (SCBH) foi de $5,7 \%$, no período de 1992 a 1998 , segundo dados fornecidos pela Comissão de Controle de Infecção Hospitalar (CCIH).

Ainda que esses dados estejam, aproximadamente, dentro dos parâmetros esperados, nos limiares recomendados pelo Center for Disease Control (CDC), de Atlanta-EUA, e mantendo-se em torno dos $5 \%$, para procedimentos cirúrgicos considerados limpos ${ }^{(2,4-5)}$, deve-se levar em conta o fato de que os pacientes submetidos às artroplastias de quadril no hospital-escola são acompanhados pela Comissão de Infecção apenas durante o período em que estão internados, segundo informação do responsável técnico por isso. Conseqüentemente, não estão incluídas em seus relatórios as infecções que, porventura, tenham se manifestado após a alta hospitalar, no ambulatório. Como os pacientes submetidos às artroplastias não são acompanhados durante um ano após a cirurgia, período em que ainda podem ocorrer infecções, tem-se a possibilidade de estar diante de índices parcialmente estimados.

Em 1994, o Center for Disease Control and Prevention (CDC-P), em Atlanta-EUA, sugeriu que os pacientes cirúrgicos devem ser acompanhados desde a cirurgia até a alta hospitalar e seguidos em ambulatório de egresso ${ }^{(6)}$. No entanto, esta parece não ser uma prática realizada nos hospitais brasileiros. 0 Serviço de Controle de Infecção Hospitalar que não possui controle de egresso cirúrgico, gera taxas de infecção subnotificadas, muito aquém da realidade ${ }^{(6-}$ 7).

A artroplastia de quadril é uma cirurgia indicada para 0 tratamento de problemas na articulação coxofemoral, como fratura, artrose, artrite reumatóide e outros, em pacientes com idade acima de 60 anos. A articulação pode ser substituída, total ou parcialmente, por uma prótese, para restabelecer sua função, promovendo 0 movimento e 0 alívio da dor.

A implantação de próteses articulares tornou-se uma cirurgia amplamente utilizada por cirurgiões no mundo inteiro, proporcionando melhor qualidade de vida aos pacientes que, anteriormente, estariam condenados ao leito. Apesar de todos os esforços e melhoria de algumas questões técnicas, essa cirurgia pode vir acompanhada de uma complicação gravíssima, a infecção.

Nas artroplastias de quadril, a infecção de sítio cirúrgico (ISC) pode ser aguda ou tardia, ocorrendo na incisão do quadril, superficial ou profundamente, por um período de até um ano no pósoperatório $^{(2,4)}$. Os implantes de próteses infectados acarretam destruição de tecidos, disfunção dos dispositivos implantados e disseminação dos patógenos ${ }^{(8-9)}$.

Há 40 anos, os índices de infecção em artroplastias de quadril giravam em torno de $13 \%{ }^{(9)}$. Atualmente, os índices norteamericanos de infecção são inferiores a $2 \%{ }^{(2,10)}$. Este decréscimo brusco nas taxas de infecção é devido às técnicas cirúrgicas e de assepsia avançadas; a um centro cirúrgico diferenciado, com salas de operação com ar ultra-limpo, fluxo laminar; equipe cirúrgica utilizando roupas com sistema próprio de exaustão de ar, e ao uso de antibioticoprofilaxia ${ }^{(8)}$. Muitos desses procedimentos e tecnologia não são encontrados na maioria dos centros cirúrgicos dos hospitais mineiros.

O desenvolvimento da infecção no local da prótese pode ocorrer de três maneiras: por implantação direta na ferida cirúrgica; por disseminação hematogênica e por reativação de uma infecção latente ${ }^{(8-9,11)}$.

As infecções de prótese são divididas em três estágios, de acordo com o seu tempo de manifestação, embora, para efeito de análise neste estudo, deva ser considerado o tempo estabelecido pelo CDC de Atlanta-EUA ${ }^{(2,8)}$.

- Estágio I: infecção superficial decorrente do hematoma periprotético que ocorre entre três e seis meses após a implantação da prótese. É resultado da contaminação direta no ato cirúrgico. Corresponde a $40 \%$ do total das infecções e pode evoluir para a infecção profunda, caso não haja tratamento. 
- Estágio II: infecção superficial ou profunda que ocorre entre seis meses e em até dois anos de pós-operatório e que é ainda decorrente da contaminação no ato cirúrgico. Representa $45 \%$ de todas as infecções.

- Estágio III: infecções profundas que ocorrem tardiamente, após dois anos de cirurgia. São decorrentes de disseminação hematogênica. Correspondem a $15 \%$ do total das infecções.

Aproximadamente $50 \%$ de todas as infecções de prótese são causadas por Estafilococos, igualmente divididos entre $0 \mathbf{S}$. aureus e $\circ$ S. epidermidis. Os outros $50 \%$ das infecções são causadas por Estreptococos, Proteus, Pseudomonas, Enterobacter e outros ${ }^{(2,11)}$.

A partir da inquietude proporcionada pelos problemas que envolvem a infecção hospitalar, surgiu o interesse em estudar os aspectos epidemiológicos das infecções de sítio cirúrgico nos pacientes submetidos a cirurgias de artroplastia de quadril, objetivando determinar a taxa de incidência de ISC, entre agosto de 1992 e outubro de 1998; identificar os possíveis fatores de risco associados à presença de ISC; determinar o tempo de manifestação da infecção, além de identificar os microorganismos responsáveis pelas infecções no sítio cirúrgico da artroplastia.

\section{MÉTODOS}

Trata-se de um estudo epidemiológico, tipo coorte e não concorrente, também designado como estudo de coorte histórico, realizado em um hospital-escola de grande porte, geral e filantrópico de Belo Horizonte-Minas Gerais.

A população estudada foi composta por todos os pacientes submetidos a artroplastias de quadril realizadas no Hospital Central da SCBH. Como não houve possibilidade de acesso a três dos 308 prontuários, a amostra do estudo foi constituída pelas informações contidas em 305 prontuários de pacientes cadastrados no banco de dados referentes ao componente cirúrgico, do Programa de Vigilância Epidemiológica, do Serviço de Controle de Infecção Hospitalar do hospital em estudo.

Para o estudo, foram selecionados os pacientes submetidos a cirurgias primárias de artro-plastia parcial e total do quadril, os quais se enquadravam nos critérios estabelecidos para a de-nominação de paciente e procedimento cirúrgico do NNISS (National Nosocomial Infections Surveillance System), propostos pelo CDC de Atlanta-EUA para a coleta dos dados; e ainda os pacientes que tivessem manifestado os sinais de infecção em um ano de pós-operatório ${ }^{(12)}$.

Analisou-se a infecção de sítio cirúrgico nas artroplastias de quadril e seus possíveis fatores de risco. A categorização da ISC baseou-se na ausência (não) ou presença (sim) da infecção, podendo esta ser incisional, superficial ou profunda.

Consideraram-se como variáveis independentes os seguintes fatores de risco à ISC, cuja presença está associada a uma maior probabilidade de que uma infecção venha a desenvolverse: potencial de contaminação da ferida cirúrgica; duração da cirurgia (período de tempo em horas e minutos desde o início do ato anestésico até o fechamento da incisão cirúrgica. Considera-se a duração esperada, para a cirurgia de artroplastia de quadril, o período de três horas); tipo de anestesia (anestesia por condução-peridural/ raquidiana e associada-geral e condução); procedimento cirúrgico (artroplastia total e parcial do quadril); condição geral do paciente: condições físicas do paciente determinadas pelo anestesista, no período pré-operatório. 0 risco cirúrgico do paciente, considerado preditor de risco de infecção, é definido em cinco categorias ou escores, de acordo com os critérios propostos pela American Society of Anestesiologists (ASA). Esse escore é assim composto: ASA I (cliente saudável), ASA II (cliente com doença sistêmica discreta), ASA III (cliente com doença sistêmica grave, com limitação de atividade), ASA IV (cliente com doença sistêmica grave, incapacitante e com ameaça à vida) e ASA V (cliente moribundo, com pequena possibilidade de sobreviver por mais de 24 horas, com ou sem cirurgia); idade; tempo de internação pré e pós-operatório; uso de antimicrobiano profilático no período trans e pós-operatório; sexo; tipo de convênio para tratamento de saúde do paciente; unidade de internação; tipo de prótese (com cimento, sem cimento e hibrida); cirurgia ortopédica prévia no sítio cirúrgico; patologia de base (descritas segundo patologias dos sistemas cardiovascular, genitourinário, endócrino, osteomuscular, hematopoiético, tegumentar, sem patologia e sem informação) e causa da cirurgia (fraturas, artrose coxofemoral, necrose avascular da cabeça do fêmur e pseudoartrose do colo do fêmur).

A maioria das variáveis com três ou mais categorias foram dicotomizadas para posterior análise estatística. Quanto à variável patologia de base, devido ao grande número de patologias e associações, um especialista sugeriu que as doenças fossem agrupadas por sistemas. Procedeu-se da mesma forma em relação à variável causa da cirurgia. Utilizou-se um instrumento para coleta de dados baseado em protocolo preestabelecido ${ }^{(13)}$, o qual sofreu adaptações e foi acrescido de algumas variáveis específicas à cirurgia de artroplastia de quadril, contemplando dados exigidos para 0 preenchimento do protocolo estabelecido pelo Sistema NNISS.

Procedeu-se à coleta de informações junto aos prontuários de pacientes submetidos a cirurgias de artroplastia de quadril, pelo Serviço de Cirurgia Ortopédica da SCBH, após a aprovação do projeto de pesquisa (Protocolo n 007/99) pela Comissão de Ética e Pesquisa do hospital, por respeitar a Resolução $n^{0} 196 / 96$ sobre pesquisa envolvendo seres humanos. 
A falta de informações nos prontuários médicos e, principalmente, aquelas referentes ao atendimento dos pacientes no ambulatório do hospital foi a maior dificuldade encontrada para se atingirem os objetivos propostos neste estudo. Em conseqüência da falta de registros do atendimento dos pacientes no ambulatório, as infecções, após a alta, detectadas neste estudo, foram aquelas que acometeram pacientes que necessitaram de uma reinternação no hospital para tratamento e compuseram parte da listagem fornecida pela $\mathrm{CClH}$ do hospital, podendo, então, ser analisadas.

Para tratamento dos dados, utilizou-se o Programa EPIINFO, versão 6.0. Os dados obtidos foram lançados em um banco de dados específico e tratados estatisticamente.

$\mathrm{Na}$ análise descritiva das variáveis numéricas, utilizou-se a distribuição de freqüências, as medidas de tendência central e as medidas de variabilidade.

Para verificar a associação entre as variáveis independentes e a infecção de sítio cirúrgico, utilizaram-se tabelas de contingência (tabelas $2 \times 2$ ), com 0 cálculo da significância da associação, pelo teste de qui-quadrado de Mantel-Haenszel $\left(X^{2}\right)$, com correção de Yates, teste exato de Fisher e teste de qui-quadrado de tendência. Para o cálculo da força desta associação, utilizou-se 0 Risco Relativo (RR), com um nível de significância de 0,05\% e intervalo de confiança (IC) de 95\%.

\section{RESULTADOS E DISCUSSÃO}

Dos 305 pacientes deste estudo, verificou-se que $63 \%$ dos pacientes foram submetidos a artroplastias totais do quadril, e $37 \%$, a artroplastias parciais. Nos dias atuais, a artroplastia total do quadril tem sido a modalidade cirúrgica de escolha dos cirurgiões, por apresentar melhores resultados em termos de durabilidade, desgaste e perda da prótese, ao relacioná-la à hemiartroplastia.

Quanto às condições clínicas dos pacientes, esses foram classificados como encontrando-se em ASA I (33\%), ASA II (52\%), ASA III (14\%), 1\% em ASA IV e nenhum paciente em ASA V, no momento das cirurgias. Pôde-se observar que mais da metade dos pacientes eram portadores de alguma doença sistêmica, mas não impeditiva para a realização da cirurgia.

Quanto ao tempo de internação pré-operatório, $67,2 \%$ dos pacientes estiveram internados até 5 dias; $25,3 \%$, de 6 a 11 dias; $4,2 \%$, entre 12 e 21 dias, e $3,2 \%$ dos pacientes, de 22 a 47 dias. A média de dias de internação foi de 5 dias; 0 desvio padrão, de 6,2 dias. Períodos prolongados de internação, antes da realização da cirurgia, têm sido associados com o aumento do risco de infecção de sítio cirúrgico ${ }^{(1)}$. Estudos mostraram que a permanência pré-operatória do paciente, acima de cinco dias, tem associação significativa com a ocorrência de ISC intra-hospitalar e após a alta, com um risco elevado, quando comparado com a permanência inferior a esse período ${ }^{(6)}$.

Quanto ao tempo de internação no período do pósoperatório, $11,5 \%$ dos pacientes estiveram internados de 1 a 7 dias; $19,2 \%$ permaneceram por 8 a 14 dias; $34,6 \%$, de 15 a 21 dias; $11,5 \%$ corresponderam aos pacientes internados entre 22 e 30 dias, e 23,1\%, de 31 a 65 dias. A média de internação foi de 11 dias, e o desviopadrão, de 9,2 dias. 0 valor mínimo de dias de internação pósoperatória foi de 1 dia, e o máximo, 65 dias.

A maioria dos procedimentos cirúrgicos $(94,4 \%)$ foram realizados sob anestesia por condução (raquidiana/peridural), 5,6\% sob anestesia associada (geral e condução), e nenhum paciente foi submetido unicamente a anestesia geral. Pode-se dizer que a anestesia por condução foi o tipo de anestesia mais empregado na realização das artroplastias de quadril.

Observou-se que, entre os pacientes do estudo, $96,7 \%$ estavam internados na clínica ortopédica, e apenas 3,3\% encontravam-se internados em outras clínicas do hospital, como a clínica geral, quimioterapia e dermatologia.

Entre os 305 procedimentos cirúrgicos analisados, detectaram-se 27 procedimentos em que ocorreram infecções no sítio cirúrgico. Consideraram-se 26 infecções, pois, em um dos casos, o tempo de manifestação da infecção (1320 dias) ultrapassou o limite de um ano, estipulado pelo procedimento NNISS, para considerarse como infecção hospitalar ${ }^{(12)}$. Portanto, a incidência de infecção acumulada foi de $8,5 \%$ para esse tipo de procedimento cirúrgico (Figura 1).

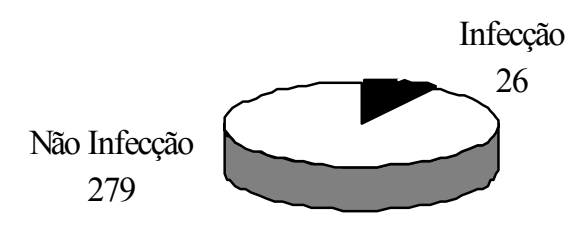

Figura 1 - Distribuição dos casos de infecção de sítio cirúrgico nas cirurgias de artroplastia do quadril. Belo Horizonte - 1992-1998

A taxa norte-americana de infecção para cirurgias de artroplastia de quadril, consideradas limpas, é inferior a $2 \%^{(8-11)}$. A taxa encontrada neste estudo está acima do preconizado pela literatura.

Dentre os 26 pacientes que contraíram infecção, em 23 $(88,5 \%)$ deles, as infecções foram categorizadas como superficiais, com uma incidência de 7,5\%, e, em $3(11,5 \%)$, como profundas, com uma incidência de $0,98 \%$.

Ainda no período de internação, foram detectadas 20 $(76,9 \%)$ infecções, todas elas superficiais. No período pós-alta, foram detectados 6 casos de infecções (23,1\%). Destes, três com infecções superficiais e três com infecções profundas. 
Chama a atenção o fato de as infecções superficiais terem sido a maioria das infecções neste estudo, todas elas manifestadas durante a internação. Este fato pode ser explicado porque, normalmente, as infecções superficiais manifestam-se mais precocemente no período pós-operatório, enquanto as infecções profundas costumam se manifestar em períodos mais tardios ${ }^{(8-9)}$. Um maior tempo de internação pós-operatória pode também ter favorecido a detecção das infecções, já que o tempo médio de internação obtido no estudo foi de 11 dias, com um tempo máximo de permanência de 65 dias, período em que a infecção superficial pode se manifestar. 0 tempo médio de manifestação da infecção hospitalar pode ser de 12 dias, o que também pode explicar uma incidência maior das infecções detectadas durante a internação ${ }^{(14)}$.

A baixa taxa de incidência de ISC, no período pós-alta (2\%), pode ser explicada pelo fato de não terem sido encontrados registros dos pacientes atendidos no ambulatório, o que impediu a deteç̧ão e controle de infecções que possam ter ocorrido nesse período.

Esses resultados podem reforçar a suspeita de subnotificação das taxas de ISC no serviço em estudo. Percebeu-se a falta de uma vigilância epidemiológica eficaz dos egressos cirúrgicos, aquela efetivada pelo acompanhamento dos pacientes após a alta hospitalar. No caso das artroplastias de quadril, a vigilância deveria estender-se a um ano após a cirurgia, período em que podem ocorrer infecções e estas serem relacionadas ao ato cirúrgico e à hospitalização.

Observou-se que, dos 20 casos de pacientes com infecção detectada durante o período de internação, 8 ocorreram nos primeiros 10 dias, e 12 deles, após 10 dias de internação. Todos os casos ocorreram dentro dos 30 dias de pós-operatório. Ressalta-se que, dos 6 casos de infecções detectadas após a alta hospitalar, 2 ocorreram no $17^{\circ}$ dia, e 4 manifestaram-se em um prazo de 6 meses.

As taxas de incidência de infecção de sítio cirúrgico anuais foram crescentes para o ano de $1992(7,7 \%)$ e de 1993 (17,3\%), e decrescentes para os anos de $1994(13,4 \%)$ e 1995 (3,2\%). Nos anos de $1996(5,1 \%$ ) e 1997 (5,6\%), as taxas foram próximas, porém superiores às encontradas no ano de 1995. Em 1998, os dados mostram uma taxa aumentada de incidência de ISC (7\%), em relação aos dois anos anteriores (Figura 2).

O ano de 1993 apresentou a maior incidência de infecção entre os pacientes submetidos a artroplastias de quadril no hospital $(17,3 \%)$.

É importante ressaltar que problemas relativos às informações contidas em prontuários dos pacientes, como a falta ou escassez de alguns dados, limitam as análises, uma vez que não permitem inferências, apenas algumas suposições. $O$ fato de se terem encontrado índices maiores de infecção relativos a procedimentos cirúrgicos de artroplastias no hospital, naquele ano, poderia, em parte, ser explicado pela mudança da Portaria 196/83 pela Portaria 930/92, que orienta as ações das Comissões para uma vigilância ativa das infecções. Além disso, pode, também, ter ocorrido um incremento das ações da CCIH-SCBH, aumento em sua eficiência e conseqüente encontro de taxas crescentes de infecção logo após o início de busca ativa das infecções ou mesmo a ocorrência de surto de infecção nesse tipo de procedimento, devido a causas variadas, como a utilização de próteses de má qualidade, técnica cirúrgica, presença de microorganismos multiresistentes ou oportunistas, resistência antimicrobiana e outros ${ }^{(2)}$.

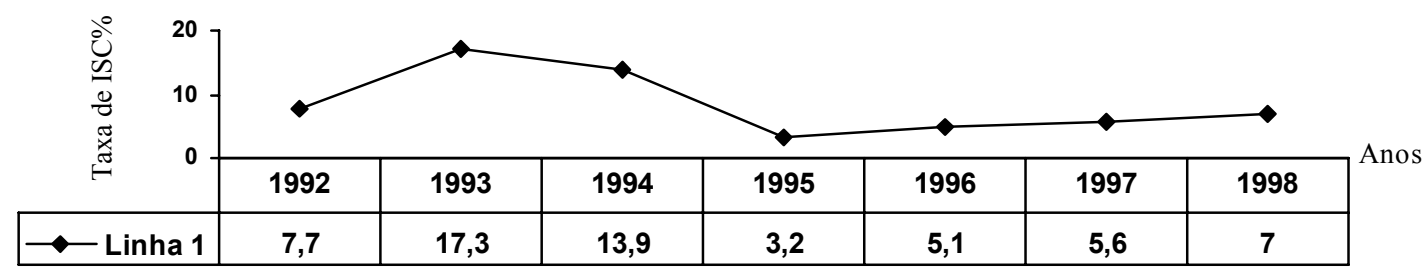

Figura 2 - Distribuição das taxas de incidência de infecção de sítio cirúrgico nas artroplastias de quadril segundo os anos. Belo Horizonte 1992-1998

Observou-se que $30,8 \%$ dos pacientes com ISC apresentavam alguma patologia de base relacionada ao sistema cardiovascular; $15,5 \%$ apresentavam patologias relacionadas ao sistema osteomuscular; 7,7\% apresentavam patologias do sistema endócrino; 7,7\%, patologias do sistema genitourinário; $11,4 \%$, referentes a outros sistemas, e 26,9\% não apresentavam nenhuma patologia de base. É certo que pacientes idosos apresentam um maior número de problemas crônico-degenerativos e, entre os problemas relacionados ao sistema cardiovascular, há a hipertensão arterial, comum em grande parte dos pacientes idosos.

Observou-se, na população estudada, a ocorrência de 10 óbitos. Dos dez pacientes que foram a óbito, cinco apresentavam infecção no sítio cirúrgico. Todos os cinco óbitos foram relacionados à infecção adquirida no hospital.

Em relação aos microorganismos isolados nas culturas das feridas cirúrgicas, o Staphylococcus aureus foi o microorganismo predominante; isolado em 11 das 15 culturas realizadas, seguido do Staphylococcus epidermidis, isolado em 3 das 15 culturas. Em 11 
prontuários, não constava resultado de cultura. Esse achado é confirmado na literatura, que afirma ser $\mathrm{o} \mathbf{S}$. aureus 0 patógeno que mais se relaciona à infecção em sítio cirúrgico onde foi inserida uma prótese de quadril ${ }^{2,9)}$.

Comportaram-se como fatores de risco as variáveis condição clínica do paciente (ASA); tempo de internação no pré e pós-operatório; tipo de anestesia; tipo de procedimento cirúrgico e unidade de internação (Tabela 1). Comportaram-se como fatores de proteção as variáveis uso de antibioticoprofilaxia no período trans e pós-operatório.

Observou-se que a maioria dos pacientes $(65,4 \%)$ que apresentaram ISC realizaram artroplastia parcial do quadril e tiveram como causa para a realização da cirurgia, algum tipo de fratura no colo do fêmur. Dos que realizaram artroplastia total do quadril e apresentaram ISC, $23,1 \%$ tiveram como causa para a cirurgia, a artrose coxofemoral; $7,7 \%$, necrose avascular da cabeça do fêmur, e $3,8 \%$, pseudoartrose do colo do fêmur.

Alguns fatores podem explicar a maior incidência de infecção entre os pacientes submetidos a cirurgia para colocação de próteses parciais: idade avançada (ocorrência de um maior número de doenças crônico-degenerativas), formação de "debris" (devido ao atrito da prótese de metal com o acetábulo), urgência do procedimento (impedindo que o paciente seja mais bem preparado para 0 ato cirúrgico) e trauma ósseo e de partes moles causado pela fratura, levando a uma formação maior de hematomas ${ }^{(9,15-16)}$. Pacientes que realizaram artroplastia parcial do quadril apresentaram um risco 3,2 vezes maior de se infectarem do que os pacientes que fizeram artroplastia total do quadril.

Tabela 1 - Distribuição das variáveis que se comportaram como fatores de risco e a ISC

\begin{tabular}{|c|c|c|c|c|c|c|c|c|c|}
\hline \multirow[t]{3}{*}{ Variá veis } & \multicolumn{4}{|c|}{ Infeç̧ão de sitio cirúrgico } & \multirow[t]{3}{*}{ Total } & \multirow[t]{3}{*}{$\mathbf{R R}$} & \multirow[t]{3}{*}{ I.C. $95 \%$} & \multirow[t]{3}{*}{$x^{2}$} & \multirow[t]{3}{*}{ Valor p } \\
\hline & \multicolumn{2}{|c|}{ Sim } & \multicolumn{2}{|c|}{ Não } & & & & & \\
\hline & $\mathbf{H}$ & $\%$ & H & $\%$ & & & & & \\
\hline \multicolumn{10}{|l|}{ Tipo Cirurgia } \\
\hline Prótese Parcial & 17 & $(65,4 \%)$ & 096 & $(34,4 \%)$ & 113 & - & - & & \\
\hline Prótese Total & 09 & $(34,6 \%$ & 183 & $(65,6 \%)$ & 192 & 3,21 & {$[1,48 ; 6,96]$} & $x^{2}=8,50$ & $p=0,00354824$ \\
\hline Total & 26 & $(100 \%)$ & 279 & $(100 \%)$ & 305 & & & & \\
\hline \multicolumn{10}{|l|}{ AS } \\
\hline । & 07 & $(27 \%)$ & 093 & $(33,5 \%)$ & 100 & 1,00 & - & & \\
\hline$\|$ & 08 & $(31 \%)$ & 151 & $(54,3 \%)$ & 159 & 0,72 & {$[0,27 ; 1,92]$} & & \\
\hline III & 08 & $(31 \%)$ & 034 & $(12,2 \%)$ & 042 & 2,72 & {$[1,05 ; 7,02]$} & $x^{2}$ de tendência & \\
\hline $\mathrm{N}$ & 03 & $(1 \%)$ & 000 & (0\%) & 003 & 14,29 & {$[6,99 ; 9,19]$} & $=11,509$ & $p=0,00069$ \\
\hline Total & 26 & $(100 \%)$ & $270^{+}$ & $(100 \%)$ & 304 & & & & \\
\hline \multicolumn{10}{|c|}{ Permanência Pré-operatória } \\
\hline até 5 dias & 09 & $(34,6 \%)$ & 196 & $(00,3 \%)$ & 205 & 1,00 & - & & \\
\hline 6-11 dias & 10 & $(30,5 \%)$ & 067 & $24,0 \%)$ & 077 & 2,96 & {$[1,31 ; 6,69]$} & & \\
\hline 12-21 dias & 02 & $(07,7 \%)$ & 011 & $(03,9 \%)$ & 013 & 3,50 & {$[0,04 ; 4,50]$} & $x^{2}$ de tendência & \\
\hline $21-47$ dias & 05 & $(19,2 \%)$ & 005 & $(01,0 \%)$ & 010 & 11,39 & {$[4,68 ; 27,24]$} & $=24,766$ & $p=0,0000$ \\
\hline Total & 26 & $(100 \%)$ & 279 & $(100 \%)$ & 305 & & & & \\
\hline \multicolumn{10}{|c|}{ Permanência Pós-operatória } \\
\hline $1-7$ dias & 03 & $(11,5 \%)$ & 128 & $(45,9 \%)$ & 131 & 1,00 & - & & \\
\hline 8-14 dias & 05 & $(19,2 \%)$ & 103 & $(36,9 \%)$ & 108 & 02,02 & {$[0,49 ; 8,27]$} & & \\
\hline $15-21$ dias & 09 & $(34,6 \%)$ & 034 & $(12,2 \%)$ & 043 & 09,14 & {$[2,59 ; 32,23]$} & $x^{2}$ de tendência & \\
\hline $22-30$ dias & 03 & $(11,5 \%)$ & 008 & $(02,9 \%)$ & 011 & 11,91 & {$[2,72 ; 52,18]$} & $=41,94$ & $p=0,0000$ \\
\hline $31-65$ dias & 06 & $(23,1 \%)$ & 006 & $(02,1 \%)$ & 012 & 21,83 & {$[6,23 ; 76,48]$} & & \\
\hline Total & 26 & $(100 \%)$ & 279 & $(100 \%)$ & 305 & & & & \\
\hline \multicolumn{10}{|l|}{ Tipo Anestesia } \\
\hline Associada & 05 & $(19,2 \%)$ & 015 & $(05 \%)$ & 020 & - & - & & \\
\hline Conduģ ẫo & 21 & $(80,0 \%)$ & 264 & $(6 \%)$ & 285 & 3,39 & {$[1,43 ; 8,05]$} & $x^{2}=5,36$ & $p=0,019339$ \\
\hline Total & 26 & $(100 \%)$ & 279 & $(100 \%)$ & 305 & & & & \\
\hline \multicolumn{10}{|c|}{ Unidade de internação } \\
\hline Outras & 04 & $(15,4 \%)$ & 006 & $(02,2 \%)$ & 010 & - & - & & Fisher \\
\hline Ortopedia & 22 & $(84,6 \%)$ & 273 & $(97,8 \%)$ & 295 & 5,03 & {$[2,27 ; 12,66]$} & $x^{2}=9,29$ & $p=0,0061862$ \\
\hline Total & 26 & $(100 \%)$ & 279 & $(100 \%)$ & 305 & & & & \\
\hline
\end{tabular}

* Um prontuário não continha informação do ASA do paciente

Nota: Os números entre parênteses são porcentagens em relação ao total da coluna 
Quanto à condição clínica e à ISC, os pacientes classificados como sendo ASA I corresponderam a 27\%; ao ASA II, $31 \%$, ambos com risco igual de infecção. Ao ASA III, corresponderam $31 \%$ dos pacientes, com um risco 2,7 vezes a mais de contraírem infecção do que os pacientes classificados como sendo ASA I. Ao ASA IV, corresponderam $11 \%$ dos pacientes que apresentaram um risco 14,3 vezes maior de contraírem infecção, quando comparados aos pacientes classificados no ASA I. Demonstrou-se que o aumento da gravidade das condições clínicas do paciente, tende a aumentar o risco de ele contrair infecção. A severidade e o tipo de doença que motivou a cirurgia ${ }^{(1-2,17)}$, assim como a presença de doenças intercorrentes, incrementam o risco de os pacientes contraírem infecção no sítio cirúrgico. Encontrou-se um $X^{2}$ de tendência igual a 11,509 , e um valor de $p$ igual a 0,00069 , demonstrando relação estatisticamente significativa entre a condição clínica do paciente e infecção.

O risco de se contrair infecção foi 11,4 vezes maior nos pacientes que estiveram internados por um período de tempo entre 22 e 47 dias, antes de serem operados, quando comparado aos pacientes que tiveram um tempo de internação pré-operatório inferior a cinco dias. Foi encontrado um $X^{2}$ de tendência igual a 24,8 e um valor de $p$ de aproximadamente zero, revelando uma associação entre a variável tempo de internação no pré-operatório e a presença de infecção, mostrando que o risco de infecção tende a aumentar à medida que aumentam os dias de internação no pré-operatório. 0 período de hospitalização pré-operatória prolongado pode provocar modificação na flora microbiana do paciente e conseqüente colonização por microorganismos presentes no ambiente hospitalar ${ }^{(13)}$. Alguns autores encontraram resultados semelhantes $e$ chegaram a uma taxa de incidência de infecção de 13,5\% para a permanência pré-operatória superior a 5 dias, e uma taxa de $6,6 \%$ para uma permanência hospitalar entre 2 e 5 dias, de pacientes submetidos a cirurgias gerais e digestivas ${ }^{(18)}$.

Pacientes que permaneceram internados após a cirurgia, por um período acima de 30 dias, tiveram um risco de contrair infecção 21,8 vezes maior do que os pacientes que ficaram internados por um período inferior a sete dias. Foi verificado um $X^{2}$ de tendência igual a 41,9 e um valor de $p$ de aproximadamente zero, demonstrando existir uma diferença estatisticamente significativa em relação ao tempo de internação pós-operatório e à infecção, mostrando que há uma tendência ao aumento do risco de contrair infecção à medida que aumentam os dias de internação no pós-operatório. 0 período de internação pós-operatório deve ser restrito ao mínimo de tempo necessário, a fim de se evitar a aquisição de contaminação pela flora microbiana hospitalar ${ }^{(16)}$.

Quanto ao tipo de anestesia empregada durante 0 procedimento cirúrgico, encontrou-se um risco relativo de 3,4 -
$[1,43 ; 8,05]$ e um valor de $p$ igual a 0,019339 , revelando uma associação do tipo fator de risco e demonstrando diferença estatisticamente significativa entre a variável tipo de anestesia e a ISC. Os pacientes submetidos a anestesia associada tiveram um risco 3,4 vezes maior de se infectarem do que os que foram submetidos a anestesia do tipo condução. Anestesias gerais deprimem mais os pacientes do ponto de vista imunológico, colocando-os mais predisponentes a adquirirem infecções.

Em um estudo ${ }^{(13)}$ sobre ISC, realizado no ano de 1997, em um hospital universitário de Belo Horizonte, constatou-se existir uma diferença significativa entre o grupo de pacientes que foi submetido a anestesia geral, sendo o índice de infecção superior ao observado nos demais grupos de pacientes submetidos a anestesias regionais (bloqueio e condução).

Em relação à associação da ISC e à unidade de internação do paciente, foi encontrado um risco relativo de 5,0 - $[2,27 ; 12,66] \mathrm{e}$ um valor de $p$ igual a 0,00661862 , revelando uma associação do tipo fator de risco e uma associação significativa entre a variável e a ISC. Os pacientes internados em outras clínicas como a clínica geral, quimioterapia e dermatologia, tiveram um risco cinco vezes maior de se infectarem do que os pacientes internados na clínica ortopédica.

Esse fato pode ser decorrente das características das demais clínicas em relação à clínica estudada. Os pacientes internados em outras clínicas evoluem de um tratamento clínico para um tratamento cirúrgico, o que demanda uma permanência mais prolongada no hospital, fato que explicaria a modificação na flora microbiana do paciente e, assim, maior predisposição à aquisição de infecção. 0 aumento no tempo de internação mostra-se como um fator de risco à infecção ${ }^{(13)}$.

A utilização da antibioticoprofilaxia durante os períodos trans e pós-operatório mostrou-se associada à presença ISC. Os Riscos Relativos de 0,31 - [0,14-0,67] e 0,18 - [0,09-0,37] respectivamente, revelaram que a associação existente é do tipo fator de proteção. Logo, a utilização de antibiótico nos períodos do trans e pósoperatório, reduziram os riscos de os pacientes submetidos a artroplastia de quadril contraírem infecção.

É grande a controvérsia em relação à utilização de antibioticoprofilaxia em cirurgia. No caso das cirurgias ortopédicas para colocação de próteses de quadril, sua utilização é bastante difundida e aceita pelos cirurgiões ortopedistas, mas, muitas vezes, utilizado de maneira aleatória.

O antibiótico profilático deve ser empregado apenas nos grandes procedimentos ortopédicos, nas cirurgias em que o tempo de exposição dos tecidos for longo, a dissecção dos tecidos for ampla e naquelas em que ocorra inserção de material estranho. As artroplastias totais do quadril e as fraturas do colo do fêmur são exemplos que justificam o emprego da antibioticoprofilaxia ${ }^{(19)}$. 
A insistência no uso de antibiótico profilático pode ser considerada uma imprudência, já que sua eficiência ainda não está plenamente comprovada, podendo levar a complicações graves, até mesmo mortais ${ }^{(16)}$.

\section{CONCLUSÕES}

Com a realização deste estudo pôde-se concluir que: - A taxa de incidência de infecção de sítio cirúrgico encontrada no estudo foi de 8,5\% (26/305), taxa superior à fornecida pela Comissão de Infecção hospitalar da SCBH, no mesmo período do estudo (5,6\%), comprovando a hipótese de subnotificação; a grande maioria das infecções encontradas foi diagnosticada durante a internação $(76,9 \%)$ do paciente no hospital. Em todos esses casos, as infecções foram categorizadas como superficiais e manifestaram-se dentro dos trinta dias de pós-operatório;

- As infecções detectadas após a alta hospitalar $(23,1 \%)$ foram aquelas em que os pacientes necessitaram de uma reinternação para tratamento e ocorreram dentro dos seis meses;

- As variáveis sexo, idade, tipo de convênio de saúde do paciente,

\section{REFERÊNCIAS BIBLIOGRÁFICAS}

1. Mangram AJ, Horan TC, Pearson ML, Silver LC, Jarvis WR. Guideline for prevention of surgical site infection. Infect Control Hosp Epidemiol 1999 April; 20(4):97-134.

2. Rodrigues MAG, Almeida GN. Infecções do sítio cirúrgico. In: Martins MA, organizadora. Manual de infecção hospitalar: epidemiologia, prevenção e controle. $2^{\text {a }}$ ed. Belo Horizonte (MG): Medsi; 2001. p.171-89.

3. Santos NQ. Infecção hospitalar: uma reflexão histórico crítica. Florianópolis: UFSC; 1997.

4. Horan TC, Gaynes RP, Martone WJ, Jarvis WR, Emori TG. CDC definitions of nosocomial surgical site infections, 1992: a modification of CDC definitions of surgical wound infections. Infect Control Hosp Epidemiol 1992;13(10):606-8.

5. Rabhae GN, Ribeiro NF, Fernandes AT. Infecção em sítio cirúrgico. In: Fernandes AT, Fernandes MOV, Ribeiro NF. Infecção hospitalar e suas interfaces na área da saúde. São Paulo (SP): Atheneu; 2000. p.479-505.

6. Oliveira AC. Controle de egresso cirúrgico: impacto na incidência da infecção de sítio cirúrgico em um hospital universitário. [Dissertação]. Belo Horizonte (MG): Escola de Enfermagem/UFMG; 1999.

7. Rodrigues EAC. Infecções hospitalares: prevenção e controle. In: Rodrigues EAC, Mendonça JS, Amarante JMB. Histórico das infecções hospitalares. São Paulo (SP): Sarvier; 1997. p.1-27. lado do quadril operado, abordagem cirúrgica, uso ou não do cimento ósseo, duração da cirurgia, classificação da ferida operatória e a cirurgia prévia no sítio cirúrgico não se comportaram como fatores de risco à ISC;

- As variáveis que se comportaram como fatores de risco de ISC foram a condição clínica do paciente (ASA), tempo de internação pré e pós-operatório; tipo de anestesia, tipo de cirurgia realizada e unidade de internação;

- As variáveis cujos riscos relativos revelaram uma associação do tipo fator de proteção, foram o uso de antibiótico no período do trans e pós-operatório;

- O microorganismo encontrado em grande parte dos casos de infecção em próteses articulares do quadril foi o Staphylococcus aureus:

- É necessário implementar a avaliação do sistema de vigilância epidemiológica das infecções de sítio cirúrgico no hospital, a fim de minimizar questões que limitaram à consecução deste estudo, como falhas no seguimento pós-alta, devido à ausência de vigilância após a alta hospitalar dos pacientes submetidos à artroplastia de quadril, e falhas de informações devido a dados incompletos ou mesmo ausentes nos prontuários de internação e ambulatório.

8. Lew DP, Waldvogel FA. Infections of skeletal prosthesis. In: Bennett JV, Brachman PS, editors. Hospital Infection. $4^{\text {a }}$ ed. Philadelphia: Lippincott-Raven; 1998. p. 613-62.

9. Yamaguti A. Infecções relacionadas a dispositivos: ortopédicos, genitourinários, mamários e oculares. In: Rodrigues EAC, Mendonça JS, Amarante JMB. Infecções hospitalares; prevenção e controle. São Paulo (SP): Sarvier; 1997. p. 209-17.

10. Fercuri R Jr. Infecção em implantes e próteses. In: Fernandes AT, Fernandes MOV, Ribeiro NF. Infecção hospitalar e suas interfaces na área da saúde. São Paulo (SP): Atheneu; 2000. p. 702-19.

11. Nafziger DA, Saravolatz LD. Infection in implantable prosthetic devices. In: Wenzel RP. Prevention and control of nosocomial infections. $3^{\mathrm{a}}$ ed. Virginia: Willians \& Wilkins; 1997. p.889-923.

12. Ministério da Saúde (BR). NNIS. Vigilância epidemiológica por componentes. Brasília (DF): Coordenação de Controle de Infecção Hospitalar; 1994.

13. Moreira LFR. Infecções de sítio cirúrgico: um enfoque epidemiológico em um hospital universitário. [Dissertação]. Belo Horizonte (MG): Escola de Enfermagem/UFMG; 1997.

14. Medeiros AM. Incidência de infecção hospitalar em pacientes com ferida cirúrgica limpa manifestada no período pós-alta. [Dissertação]. São Paulo (SP): Escola Paulista de Medicina;1993.

15. Roberts JR, Mock DJ. Joint infections. In: Betts RF, Rose RE, editors. A practical approch to infectious diseases. $4^{\text {a }}$ ed. New York: Little Brown; 1996. p. 575-605. 
16. Hungria JSF. Infecções osteomusculares: tratamento racional baseado na patologia. In: Hungria JSF. Infecções em próteses articulares. São Paulo (SP): Sarvier; 1992. p.206-13.

17. Pereira MS, Moriya TM, Gir E. Infecção hospitalar nos hospitais escola: uma análise sobre seu controle. Rev Latino-am Enfermagem 1996 janeiro; 4(1):145-62.
18. Vegas AA, Jodra VM, Soriano RL, Gil A, Garcia ML. Infeccion de la herida quirúrgica: factores de riesgo y modelo predictivo. Med Clín 1993;100(14):521-5.

19. Sandoval FA. Uso profilático de antibióticos em cirurgia ortopédica. Rev Bras Ortop 1987 junho; 22(5):152-4. 